\title{
PLATAFORMA BRASIL E OS DESAFIOS PARA \\ PESQUISADORES DA ÁREA DE SAÚDE QUE REALIZAM \\ PESQUISA QUALITATIVA
}

\author{
BRAZIL PLATFORM AND THE CHALLENGES FOR HEALTH \\ RESEARCHERS WHO CONDUCT QUALITATIVE RESEARCH
}

\author{
Luciana Puchalski Kalinke ${ }^{1}$ \\ Rafaela Gessner Lourenço ${ }^{2}$
}

\begin{abstract}
Resumo: Este estudo objetiva refletir sobre os principais entraves relacionados as diretrizes éticas que pesquisadores qualitativos brasileiros, da área de saúde, encontram para os registros de suas pesquisas. Foram analisadas as Resoluções do Conselho Nacional de Saúde sobre o tema, o Sistema Nacional de Informações sobre Ética em Pesquisa e a Plataforma Brasil. A despeito do avanço nas publicações das diretrizes éticas de pesquisas com seres humanos que propiciaram suprir lacunas sobre as especificidades de algumas áreas e populações, percebe-se que o caráter voltado à pesquisa experimental e biomédica permeia o processo de registro das pesquisas na Plataforma Brasil, o que resulta em desafios para os pesquisadores qualitativos, como: a definição da amostra, do número de participantes e a inserção dos riscos e benefícios da pesquisa. Faz necessário que o sistema seja reformulado a fim de respeitar as especificidades de outras naturezas de pesquisas desenvolvidas na área da saúde.
\end{abstract}

Palavras-chave: Pesquisa Qualitativa; Ética em Pesquisa; Projetos de Pesquisa; Pesquisa em Saúde.

\begin{abstract}
This study aims to reflect on the main obstacles related to the ethical guidelines that Brazilian qualitative researchers in the health area encounter when registering their research. We analyzed the Resolutions of the National Health Council on the subject, the Sistema Nacional de Informações sobre Ética em Pesquisa (SISNEP) and Plataforma Brasil. Despite the advance in the publication of ethical guidelines for research with human beings, which allowed for the filling of gaps on the specificities of some areas and populations, it is perceived that the character of experimental and biomedical research permeates the process of registering research in Plataforma Brasil, which results in challenges for qualitative researchers, such as the definition of the sample, the number of participants, and the inclusion of the risks and benefits of research. It is necessary that the system be reformulated to respect the specificities of other types of research developed in the health area.
\end{abstract}

Keywords: Qualitative Research; Research Ethics; Research Projects; Health Research.

\section{Introdução}

Submeter a pesquisa qualitativa às diretrizes de uma área pragmática e sistemática, como a da pesquisa biomédica, pode se configurar como um desafio para muitos pesquisadores. Esse desafio relaciona-se ao: desenvolvimento de uma pesquisa a partir de uma abordagem interpretativa e naturalista do mundo, que estuda fenômenos em seu

\footnotetext{
${ }^{1}$ Doutorado em Ciências da Saúde pela Pontifícia Universidade Católica do Paraná (PUC/PR). Professora Associada do Departamento de Enfermagem da Universidade Federal do Paraná (UFPR), Curitiba, PR, Brasil. E-mail: lucianakalinke@ufpr.br

${ }^{2}$ Doutorado em Enfermagem pela Universidade de São Paulo (USP). Professora adjunta do Departamento de Enfermagem da Universidade Federal do Paraná (UFPR) da área de Saúde Coletiva. E-mail: rafaelagessner@ufpr.br
} 
ambiente natural e busca interpretar seus significados; ao próprio investigador ser, ou desenvolver o instrumento de coleta de dados; a impossibilidade de precisar o número de participantes a serem investigados; e do fato de as práticas interpretativas dependerem sobremaneira do pesquisador. Esta constatação gera reflexões que serão discutidas ao longo deste texto.

A pesquisa qualitativa busca, em última instância, contribuir para o avanço da ciência, da técnica e para o cuidado de pessoas, grupos e coletividades (MINAYO, 2017). Além disso, especificamente na área da saúde, destaca-se a potencialidade das investigações qualitativas no processo de transferência do conhecimento, ou seja, permitir que o resultado das investigações e produções científicas sejam utilizados no cenário da prática em saúde. Por isso, seu escopo de atuação mostra-se tão abrangente nesse campo, o que vem sendo discutido pela literatura (BAIXINHO; PRESADO; RIBEIRO, 2019).

Em que pese a diversidade de técnicas de coleta e análise de dados, a utilização de softwares voltados à pesquisa qualitativa e a adoção de ferramentas e checklists de verificação da estrutura, eficiência e transparência metodológica de trabalhos científicos qualitativos (COSTA; SOUZA, 2017), ainda persistem dificuldades para a realização desta natureza de pesquisa. Como por exemplo, o seu desenvolvimento e registro à luz das diretrizes éticas, que foram forjadas por concepções tradicionalistas de investigação, sustentadas no modelo biomédico e positivista (BAIXINHO; PRESADO; RIBEIRO, 2019).

Faz-se necessário, portanto, relembrar que, principalmente na área biomédica, para que ocorresse o desenvolvimento científico, inúmeras pesquisas foram desenvolvidas com padrões de ética e qualidade técnica discutíveis. Os abusos e conflitos éticos gerados, originaram uma série de recomendações para a condução de pesquisas em seres humanos. A elaboração de diretrizes éticas tanto no Brasil quanto no mundo, visam a garantir o respeito e a proteção dos participantes de pesquisa enquanto seres biopsicossociais e contribuírem para a efetivação da democracia deliberativa (LOPES JÚNIOR, 2016).

No contexto das especificidades que pesquisadores qualitativos encontram para a realização de suas pesquisas, emergiu a questão norteadora desta reflexão: Quais as dificuldades que os pesquisadores qualitativos da área da saúde encontram para seguir as obrigatoriedades das diretrizes que constam nas resoluções de ética em pesquisas com seres humanos no Brasil? Para respondê-la esta reflexão teve como objetivo: refletir sobre 
os principais entraves relacionados as diretrizes éticas, que pesquisadores qualitativos brasileiros, da área de saúde, encontram para os registros de suas pesquisas.

Ao considerar o dever ético e moral associado ao desenvolvimento de conhecimento produzido por meio de pesquisas qualitativas - especialmente aquelas comprometidas com a transformação social (EGRY; FONSECA, 2018) - apresenta-se a relevância deste estudo: problematizar algumas das dificuldades enfrentadas pelos pesquisadores que conduzem estudos qualitativos no que diz respeito à observância dos trâmites éticos para o registro dos seus projetos no Brasil e propor sugestões com vistas a superar essa problemática.

\section{Desenvolvimento}

Este estudo possui caráter teórico-reflexivo, foi desenvolvido em agosto de 2021 com fundamentação baseada na formulação discursiva acerca da temática, sustentada na literatura científica nacional e internacional, com análise crítica dos autores. Para construção teórica desta reflexão, foram analisadas as Resoluções do Conselho Nacional de Saúde, atuais e anteriores, (n¹96/96 e n466/12), assim como o Sistema Nacional de Informações sobre Ética em Pesquisa (SISNEP) e Plataforma Brasil. Após, a análise dos documentos, os autores discutiram os entraves que os pesquisadores qualitativos, da área de saúde, enfrentam no desenvolvimento de suas pesquisas.

\subsection{A necessidade da legislação de pesquisa com seres humanos}

A necessidade de normas oficiais que regulamentassem a pesquisa em seres humanos foi evidenciada após os abusos dentro e fora dos campos de concentração, durante a II Guerra Mundial em 1947. O Código de Nuremberg é considerado um dos primeiros documentos que garante, entre outros pontos, o consentimento voluntário do indivíduo para a participação de pesquisa, prezando pelo princípio da autonomia (BARBOSA et al., 2011). No entanto, mesmo após a sua publicação e extensa divulgação, as atrocidades com seres humanos que participavam de pesquisas ainda aconteciam.

Nas décadas de 50 e 60 , muitas pesquisas conduzidas de forma eticamente inadequada, foram publicadas em revistas científicas, o que motivou a discussão e revisão do Código de Nurenberg na $18^{\mathrm{a}}$ Assembleia da Associação Médica Mundial, em 1964. Nesta mesma Assembleia foi aprovada a $1^{\text {a }}$ Declaração de Helsinque, que introduzia a necessidade de revisão dos protocolos de pesquisa com seres humanos por comitês 
independentes. A denominação Declaração de Helsinque foi mantida nas versões posteriores realizadas em Tóquio (1975), Veneza (1983), Hong Kong (1989), Somerset West (1996), Edinburgo (2000), Seul (2008) e (Fortaleza) 2013. Destaca-se que o Brasil não é signatário da versão de 2013, por discordar da flexibilização no uso de placebo em experimentos realizados em participantes brasileiros (PASSINI, 2018).

Em relação ao Brasil, a Resolução do Conselho Nacional de Saúde (CNS) de $1^{\circ}$ de junho de 1988 (CNS n01/88), foi um dos primeiros documentos a pontuar as questões éticas em relação à pesquisa com seres humanos e apresentar normas para a condução de estudos experimentais. Possivelmente este seja um dos primeiros documentos que determinou a tendência das resoluções de pesquisa no Brasil a abordarem o caráter experimental das investigações, uma vez que pesquisas de caráter qualitativo, não eram abordadas nele.

Devido o pouco impacto da resolução CNS n ${ }^{\circ}$ 01/88 e da crescente entrada de estudos clínicos no Brasil, em 1995, foi criado um Grupo Executivo de Trabalho (GET), visando a revisar as normas de pesquisa, estabelecer e obrigar a análise bioética nos projetos de pesquisa no país. A partir dos trabalhos deste grupo, surgiu a Resolução CNS n $196 / 96$, publicada no Diário Oficial da União em 10 de outubro de 1996 (BRASIL, 1996). A Resolução CNS no 196/96, foi um marco no registro de pesquisas no Brasil, possivelmente, além dos seus princípios fundamentais, a proposta do governo era ter um banco de dados para acompanhamento das pesquisas no país. No entanto, não era possível imaginar a dimensão e a quantidade de investigações que eram desenvolvidas, o que culminou em discussões e contestações de pesquisadores de diversas áreas.

Com a Resolução no 196/96, foram instituídos os Comitês de Ética em Pesquisa (CEPs), vinculados às instituições de pesquisas, exigindo uma composição multidisciplinar (não mais que metade dos membros pertencentes a mesma profissão), incluindo, obrigatoriamente, um representante dos usuários. Foi criada também a Comissão Nacional de Ética em Pesquisa (CONEP), órgão máximo da área, ligado ao CNS, e por conseguinte ao Ministério da Saúde (MS) (BRASIL, 1996).

A CONEP é uma instância colegiada, de natureza consultiva, educativa e formuladora de diretrizes e estratégias no âmbito do Conselho, além disso, possui como premissa ser independente de influências corporativas e institucionais. Assim como os CEPs, mantém composição multidisciplinar, contando com um representante dos usuários. Seu objetivo é a avalição dos aspectos éticos das pesquisas que envolvem seres humanos, e cabe a ela avaliar e acompanhar os protocolos de pesquisa em áreas temáticas 
especiais como: genética e reprodução humana; novos equipamentos; dispositivos para a saúde; novos procedimentos; população indígena; projetos ligados à biossegurança e com participação estrangeira (BRASIL, 1996).

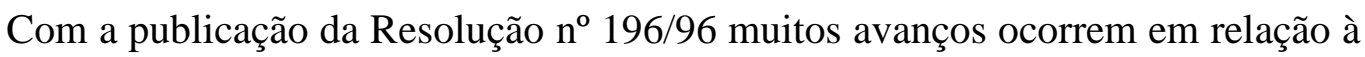
pesquisa no Brasil, principalmente quanto aos registros. A criação de CEPs foi uma das primeiras necessidades que a Resolução estabelecia. Os obstáculos operacionais, devido à falta de experiência dos membros que compunham o CEP, foram paulatinamente sanados e, em decorrência disso percebeu-se um aumento da massa crítica de pessoas para essa função, com consequente crescimento qualitativo e quantitativo dos CEPs no Brasil (FILHO, 2007).

Ao relacionarmos os campos das pesquisas qualitativas, com as premissas da Resolução no 196/96, é possível observar que elas convergem, quase que somente em seu preambulo, com destaque para o seguinte parágrafo: "Esta Resolução incorpora, sob a ótica do indivíduo e das coletividades, os quatro referenciais básicos da bioética: autonomia, não maleficência, beneficência e justiça, entre outros, e visa assegurar os direitos e deveres que dizem respeito à comunidade científica, aos sujeitos da pesquisa e ao Estado" (BRASIL, 1996, p.1). No entanto, em todo o corpo descritivo, não há informações, ou mesmo termos relacionados à pesquisa qualitativa. A Resolução é voltada a pesquisas com abordagem experimental, apresentando termos, definições e classificações específicas da área biomédica.

Com a publicação de resoluções complementares observou-se uma tentativa de suprir lacunas sobre as especificidades de algumas áreas e populações. No entanto, apesar dessas mudanças, o conceito de pesquisa ainda não se aproxima das pesquisas de natureza qualitativa, uma vez que a caracterização experimental permeia todas as resoluções complementares, como as: $n^{\circ} 240 / 97$, que define a participação de usuários nos CEPs; $n^{\circ}$ 251/97, sobre novos fármacos, medicamentos, vacinas e testes de diagnósticos; n 292/99, a respeito de pesquisas coordenadas com participação estrangerias e remessa de material biológico para o exterior, revogada pela $\mathrm{n}^{\circ} 346 / 05$, que versa sobre estudos multicêntricos; $n^{\circ}$ 300/00 a respeito de pesquisas sobre reprodução humana; $n^{\circ}$ 304/00 para pesquisas sobre população indígenas; $n^{\circ}$ 340/04 acerca de pesquisas sobre genética humana; $n^{\circ}$. 347/05 que regulamenta o armazenamento e a utilização de material biológico no âmbito da pesquisa, até a publicação da resolução atual nº . 466/12,

Em 2011, a Resolução nº 196/96 foi colocada em consulta pública, na tentativa de atualizá-la e tentar dirimir incoerências com os demais tipos de pesquisa. Assim, em 
setembro de 2012 um novo GET, em conjunto com representantes de CEPs, elaboraram um documento que deu origem a Resolução no 466/12, revogando a n 196/96.

A Resolução nº 466/12 trouxe inovações no conteúdo da norma, com ênfase no âmbito bioético dos estudos, ao enfatizar a necessidade de valorizar "o respeito pela dignidade humana e pela especial proteção devida aos participantes das pesquisas envolvendo seres humanos". Entre os novos itens incorporados, ela destaca que ao final do estudo deve ser assegurado a todos os participantes, pelo pesquisador, acesso gratuito e por tempo indeterminado, aos melhores métodos profiláticos, diagnósticos e terapêuticos que se demonstrarem eficazes (CREMESP, 2020). Essa situação não era prevista explicitamente na Resolução n 196/96.

Outro destaque que a Resolução $n^{\circ}$ 466/12 incorpora é a garantia que o medicamento em estudo possa ser oferecido por meio de estudo de extensão, de acordo com análise devidamente justificada do médico assistente do participante. Entre outras inclusões dessa Resolução, está a informação de que as participantes em idade fértil que se declararem isentas do risco de gravidez (por não exercerem práticas sexuais ou por exercerem de forma não reprodutiva) não serão obrigadas a usarem contraceptivos (BRASIL, 2012).

Além disso, essa Resolução $n^{\circ} 466 / 12$ explicita que ao explicar a pesquisa, o pesquisador deve prestar todas as informações necessárias, em linguagem clara e acessível, utilizando-se das estratégias mais apropriadas à cultura, faixa etária, condição socioeconômica e autonomia dos convidados a participar da pesquisa; concedendo o tempo adequado para que o indivíduo possa refletir, consultando, se necessário, seus familiares ou outras pessoas capazes de ajudá-lo na tomada de decisão livre e esclarecida (CREMESP, 2020). Entretanto, apesar dos ajustes, prevalece na Resolução nº 466/12 as características das pesquisas desenvolvidas, historicamente, na área biomédica.

Diante do exposto é possível analisar que o processo de avanço nas diretrizes éticas de pesquisas com seres humanos, realizado no Brasil, é amparado em um modelo biomédico de se fazer pesquisa, resultando em um conflito com o modo de fazer pesquisa para os pesquisadores qualitativos, tanto na área da saúde, quanto das Ciências Humanas e Sociais $(\mathrm{CSH})$.

Após anos de discussão, em 06 de abril de 2016 foi criada a Resolução relativa à avaliação da ética em pesquisa nas CHS. A Resolução $n^{\circ}$ 510/16 foi elaborada por representantes das associações científicas que tinham assento no GET para sua 
elaboração. Pesquisadores da área, destacam um trabalho árduo, de luta continua e exaustiva contra as resistências, preconceitos e vícios da CONEP (DUARTE, 2017).

No estudo desenvolvido por Duarte (2017) é possível conhecer a cronologia da luta da área das CHS diante das resoluções de ética em pesquisa no Brasil. Em 2001 há o registro da primeira reclamação oficial da Associação Brasileira de Antropologia ao CNS, que considerou abusiva as normas para pesquisas com a população indígena. De 2001 até 2010 várias manifestações foram realizadas por pesquisadores e instituições da área e a partir de 2011 com o fortalecimento do movimento e por meio de discussões realizadas em fóruns e eventos fomentou-se a elaboração de documentos e moções dirigidas ao MS, e outras instancias para que fossem respeitadas as especificidades das pesquisas empreendidas nas áreas de CHS.

Nesse contexto, a publicação da Resolução $n^{\circ}$ 510/16 foi atribuída como uma vitória para os representantes de associações cientificas com assento no GET. No entanto, a incompletude de aspectos fundamentais do novo sistema de avaliação da ética em pesquisa nas CHS impede que essas pesquisas rendam todo o seu potencial (DUARTE, 2017). Esse debate conduz à reflexão sobre a necessidade de alterações no processo de registro pela Plataforma Brasil dos componentes éticos das pesquisas desenvolvidas no âmbito da CHS.

\subsection{Sistemas de Informação para Pesquisas com Seres Humanos}

Apesar dos avanços registrados nos 17 anos de intervalo entre a publicação da Resolução nº196/96 para a n 466/12, o conceito de pesquisa ainda está voltado à pesquisa realizada em seres humanos, e não com seres humanos. Emerge a característica da pesquisa experimental, ou da pesquisa biomédica, e esta característica também é identificada nos sistemas de informação de pesquisa, como verificado no extinto SISNEP e, agora, na Plataforma Brasil.

O SISNEP, que posteriormente foi substituído pela Plataforma Brasil, permitiu o avanço dos registros de pesquisas no Brasil. Foi o primeiro sistema de informação, via internet, sobre pesquisas que envolvem seres humanos, possibilitando acesso a essa ferramenta para os cientistas, CEPs, CONEP e a população em geral. Entretanto, suas características eram tendenciosas às pesquisas experimentais, mais especificamente a estudos clínicos com novas drogas, deixando de lado, as características e subjetividades de outros tipos de pesquisa. 
Com o crescente número de registros de pesquisa em seres humanos, houve necessidade de maior detalhamento e melhor acompanhamento, em tempo real, das pesquisas que eram desenvolvidas no país. Assim, a partir de 2008 iniciou-se a discussão de um novo sistema para o registro de pesquisa em seres humanos, e em 15 de janeiro de 2012 o SISNEP foi desativado e substituído pela Plataforma Brasil.

A Plataforma Brasil é formada por um banco de dados com quatro fontes primárias: pesquisadores, CEP, CONEP e o público em geral. Desde seu lançamento em 2012, ela tem como objetivo criar uma interação com agências regulatórias, instituições de fomento à pesquisa, instituições internacionais e editores científicos, entretanto esta etapa ainda permanece na fase de concepção (ARAUJO; FRANCISCO, 2016).

A Plataforma permite que as pesquisas sejam acompanhadas em seus diferentes estágios, da submissão até a aprovação final pelo CEP e pela CONEP, quando aplicável, permite também, o envio de relatórios parciais e finais das pesquisas. Um ponto forte a ser destacado na Plataforma, é a possibilidade da apresentação de documentos no formato digital, o que diminui o tempo de trâmite dos projetos em todo o sistema CEP/CONEP, assim como, proporciona a sociedade, acesso aos dados públicos das pesquisas aprovadas. Aos pesquisadores e sua equipe, o sistema possibilita acesso aos dados do estudo, submetidos ao CEP e/ou CONEP (CNS, 2019). Entretanto, apesar dos avanços associados à implementação da Plataforma Brasil no que diz respeito à avaliação dos componentes éticos das pesquisas, seus objetivos não levam em conta as especificidades de algumas pesquisas desenvolvidas na área da saúde e das CHS.

Para que ocorra o registro de um projeto de pesquisa na Plataforma Brasil, é necessário seguir seis etapas: a primeira é o cadastro de todos os membros da pesquisa na Plataforma. Na segunda etapa do registro, deve-se indicar a área temática especial, quando é possível encontrar 18 itens destinados a especificidades de pesquisa em genética, reprodução humana, populações indígenas, novos equipamentos e procedimentos terapêuticos invasivos. Ao identificar esta especificidade da área temática especial, surge o questionamento: se há possibilidade do detalhamento de uma determinada área, por que não é possível respeitar o detalhamento das demais áreas de conhecimento? O formato atual, por si só, mostra o quanto o sistema é direcionado à pesquisa experimental e excludente em relação a outras modalidades de pesquisa.

Para a área da Enfermagem, desde a implementação da Plataforma Brasil percebeu-se um avanço, ainda que baseado também na característica da pesquisa experimental. Quando houve a atualização da versão da Plataforma, foi incluído no item 
"propósito principal do estudo" a descrição: Supportive Care - cuidados de enfermagem para prevenir, controlar e evitar condições clínicas do paciente. Antes desta inclusão, o sistema não tinha especificidade nenhuma da área, ou seja, o que a área de Enfermagem pesquisava não possuía registro na Plataforma. Empiricamente, os pesquisadores da Enfermagem procuravam se encaixar em algo que entendiam ser o mais próximo possível do que estavam pesquisando.

A medida em que o pesquisador avança no processo de registro do seu projeto de pesquisa na Plataforma Brasil, novos desafios surgem. Na página três, destinada ao registro da pesquisa, observa-se que aproximadamente $80 \%$ das informações solicitadas, estão destinadas à pesquisa experimental ou de intervenção. E mais uma vez, é possível questionar: se a maioria das pesquisas registradas no sistema CEP/ CONEP não tem característica experimental, por que devemos utilizar para registro uma Plataforma que foi desenvolvida com foco central no desenvolvimento de pesquisas experimentais?

A quarta etapa de registro na Plataforma é destinada ao detalhamento do estudo, nesta são solicitadas informações relacionadas ao projeto de pesquisa. Observa-se que é obrigatório o preenchimento de todos os itens da página. No terceiro item dessa etapa, surge o campo hipótese, primeiro entrave para muitos pesquisadores, em especial os qualitativos, cujos desenhos não são aplicáveis esta questão. Outro item, refere-se ao número de participantes da pesquisa, e mais uma vez, colocam-se barreiras para evoluir no registro, pois como defini-lo em certos tipos de pesquisas, principalmente as qualitativas?

Entre as pesquisas qualitativas desenvolvidas na área de saúde, pode-se citar a Teoria Fundamentada em Dados (TFD) ou Grounded Theory (GT), uma das mais utilizadas na Enfermagem nas últimas décadas (LEITE et al., 2012). Santos (2016, p.2) destaca que o foco da TFD: "é compreender as experiências e interações de pessoas inseridas em um determinado contexto social, buscando evidenciar estratégias desenvolvidas diante de situações vivenciadas [...]” e que a proposta da TFD “[...] centrase, na ação-interação humana, tornando-a um referencial metodológico relevante para a área da enfermagem e saúde, cujas práticas baseiam-se nas interações constantes entre pacientes, familiares e equipe de trabalho [...]”. Diante desta concepção e características de pesquisa é pertinente refletir sobre como submeter um projeto de pesquisa, cujo método proposto é a TFD, a um formato de registro tão engessado como o da Plataforma Brasil? 
Entraves como estes surgem a cada etapa de registro de pesquisas qualitativas na Plataforma. Muitos pesquisadores qualitativos, acabam por inserir dados imprecisos sobre a sua pesquisa, para que possam avançar as etapas no sistema. Com isto, podemos pensar e questionar a respeito da contradição ética que se estabelece ao serem inseridas informações deturpadas ou imprecisas para que a aprovação do projeto de pesquisa seja viabilizada.

Dessa maneira, para que a inserção de dados provenientes de projetos qualitativos na Plataforma Brasil seja mais acurada é necessário que as dificuldades apontadas por este estudo sejam sanadas, com destaque para: a necessidade de definição da amostra; definição do número de participantes; a inserção dos riscos e benefícios da pesquisa. Além disso, faz-se necessário que os membros dos Comitês de Ética em Pesquisa compreendam as especificidades da pesquisa qualitativa.

\section{Considerações finais}

Esta reflexão discutiu que o atual formato do sistema de informação para registro dos projetos de pesquisa no Brasil não respeita, integralmente, as especificidades de outras pesquisas desenvolvidas na área da saúde que não sejam experimentais. Porém, sabe-se que essa reflexão não é nova e acompanha outras discussões apresentadas por outras áreas do conhecimento, que, entendem ser ainda mais prejudicadas que a área da saúde no que diz respeito ao registro de seus projetos de pesquisa. É importante destacar que os pesquisadores qualitativos não querem deixar de submeter seus projetos de pesquisa a apreciação dos CEPs, no entanto, é necessário que processos sejam revistos a fim de abranger as especificidades dessa natureza de pesquisa.

\section{Referências}

ARAUJO, N.; FRANCISCO, D. Ética em pesquisa com seres humanos na web: o caso da Plataforma Brasil. Informação \& Informação, Londrina, v. 21, n.3, p.361-375, 2016.

Disponível em: pdf_bfc02e5997_0000023062.pdf (brapci.inf.br). Acesso em: 25 ago. 2021.

BAIXINHO, C.L.; PRESADO, M.H.; RIBEIRO, J. Investigação qualitativa e transformação da saúde coletiva. Ciência \& Saúde Coletiva, Rio de Janeiro, v. 24, n. 5, p.1582-1582, 2019. Disponível em: https://doi.org/10.1590/1413-81232018245.05962019. Acesso em: 31 ago.2021.

BARBOSA, S. A.; BOERY, R. N. S. de O.; BOERY, E. N.; FILHO, D. L. G.; SENA, E. L. da S.; OLIVEIRA, A. A. DA S. A Resolução 196/96 e o sistema brasileiro de revisão ética de pesquisas envolvendo seres humanos. Revista Bioética, Brasília, v.19, n.2, p. 543-521, 2011. Disponível em: https://revistabioetica.cfm.org.br/index.php/revista_bioetica/article/view/642. Acesso em: 28 ago. 2021. 
BRASIL. Plataforma Brasil será lançada dia 15. Conselho Nacional de Saúde. Brasília, 9 dez. 2009. Disponível em:

http://conselho.saude.gov.br/ultimas_noticias/2009/09_dez_plataforma_brasil.htm. Acesso em: 25 ago. 2021.

BRASIL. Ministério da Saúde. Conselho Nacional de Saúde. Resolução no 196, de 10 de outubro de 1996. Diretrizes e normas regulamentadoras de pesquisa envolvendo seres humanos. Brasília, 1996. Disponível em:

https://bvsms.saude.gov.br/bvs/saudelegis/cns/1996/res0196_10_10_1996.html. Acesso em: 27 ago. 2021.

BRASIL. Ministério da Saúde. Conselho Nacional de Saúde. Resolução no 466, de 12 de dezembro de 2012. Diretrizes e normas regulamentadoras de pesquisa envolvendo seres humanos. Brasília, 2012. Disponível em:

https://bvsms.saude.gov.br/bvs/saudelegis/cns/2013/res0466_12_12_2012.html. Acesso em: 27 ago. 2021.

COSTA, A. P.; de SOUZA, F. N. Critérios de construção e avaliação de artigos em investigação qualitativa (CCAAIQ). In: COSTA, A.P.; SÁNCHEZ-GOMEZ, M.C.; CILLEROS, M.V.M. (org.). A prática na Investigação Qualitativa: exemplos de estudos. Aveiro: Ludomedia, 2017, p.12-20. Disponível em: https://ludomedia.org/publicacoes/a-pratica-na-investigacaoqualitativa-exemplos-de-estudos/. Acesso em: 31 ago. 2021.

CREMESP: Pesquisas com seres humanos. Centro de Bioética do CREMESP. São Paulo, 27 jan. 2021. Disponível em: http://bioetica.org.br/?siteAcao=Destaques\&id=188. Acesso em: 26 de agosto de 2021.

DUARTE, L. F. D. Cronologia da luta pela regulação específica para as Ciências Humanas e Sociais da avaliação da ética em pesquisa no Brasil. Práxis Educativa, Ponta Grossa, v. 12, n. 1, p. 267-286, jan./abr. 2017. Disponível em:

http://www.revistas2.uepg.br/index.php/praxiseducativa. Acesso em: 27 de agosto de 2021.

EGRY, E. Y.; FONSECA, R.M.G.S. On the quality of qualitative research in nursing. In: COSTA, A. P. et al. (org.). Computer supported qualitative research. Cham: Springer, 2018. p.34-59. Disponível em: https://doi.org/10.1007/978-3-319-61121-1. Acesso em: 31 ago. 2021.

LEITE, J. L.; SILVA, L. J. da.; OLIVEIRA, R. M. P. de.; STIPP, M. A. C. Reflexões sobre o pesquisador nas trilhas da Teoria Fundamentada nos Dados. Rev. Esc. Enferm. USP, São Paulo, v. 46, n. 3, p. 772-777, 2012. Disponível em: https://doi.org/10.1590/S008062342012000300033. Acesso em: 28 ago. 2021.

MARQUES, F. J. Ética em pesquisa: dez anos da resolução CNS 196/96. Rev. Bras. Reumat., São Paulo, v. 47, n. 1, p. 2-3, 2007. Disponível em: https://doi.org/10.1590/S048250042007000100002. Acesso em: 28 ago. 2021.

MINAYO, M.C.S. Profundas raízes de uma árvore frondosa: fundamentos e diversidade em pesquisa qualitativa. In: COSTA, A.P.; SÁNCHEZ-GOMEZ, M.C.; CILLEROS, M.V.M. (org.). A prática na Investigação Qualitativa: exemplos de estudos. Aveiro: Ludomedia, 2017, p.5-11. Disponível em: https://ludomedia.org/publicacoes/a-pratica-na-investigacaoqualitativa-exemplos-de-estudos/ Acesso em: 31 ago. 2021. 
MONTEIRO, P.V. et al. Quando cuidar do corpo não é suficiente: a dimensão emocional do cuidado de enfermagem. Rev Min Enferm., Belo Horizonte, v. 20, s/n, p. e957, 2016. Disponível em: http://www.dx.doi.org/10.5935/1415-2762.20160026. Acesso em: 28 ago. 2021.

PASSINI, R.; PINHEIRO, M.; MONTAGNER, M. Declaração de Helsinque: flexibilização do uso do placebo, um interesse do mercado farmacêutico. Rev. Bras. Bioética, Brasília, v. 14, n. e, p. 1-20, 2018. Disponível: https://doi.org/10.26512/rbb.v14i0.14687. Acesso em: 27 ago. 2021.

SANTOS, J. L. G. et al. Perspectivas metodológicas para o uso da teoria fundamentada nos dados na pesquisa em enfermagem e saúde. Escola Anna Nery, Rio de Janeiro, v. 20, n. 3, p. e20160056, 2016. Disponível em: https://doi.org/10.5935/1414-8145.20160056. Acesso em: 28 ago. 2021.

Convite feito em: 10 de maio de 2021

Revisão feita em: 20 de novembro de 2021 